http://dx.doi.org/10.21611/qirt.2017.016

\title{
NONCONTACT CONCRETE CRACK EVALUATION TRHOUGH HYBRID IMAGE SCANNING
}

\author{
Yun-Kyu An ${ }^{\dagger *}$, Keun-Young Jang \\ Sejong University, Seoul, 05006, South Korea \\ +*Presenting and Corresponding Author: yunkyuan@sejong.ac.kr
}

\begin{abstract}
This paper presents a new hybrid image scanning technique for concrete crack evaluation. The hybrid image combining vision and infrared (IR) thermography images can improve crack detectability as well as reduce false alarms. The hybrid image scanning system is developed by integrating vision camera, IR camera, continuous-wave (CW) laser and mechanical scanning jig. The CW laser continuously generates thermal waves, and the vision and IR cameras simultaneously record the corresponding vision and IR images on the region of interests (ROI) of a target structure. By spatially scanning the hybrid images with the limited field of view (FOV) using the scanning jig, even large ROI can be effectively inspected. Then, the corresponding image processing algorithm including the distortion calibration and image conversion to spatially integrated FOV images is proposed. The proposed system and algorithm are experimentally validated using a lab-scale concrete specimen with artificial macro-cracks $(\geq 500 \mu \mathrm{m})$, micro-cracks $(<500 \mu \mathrm{m})$ and a fake crack. The experiment results reveal that the hybrid image scanning technique successfully visualizes various macro- and micro-cracks, and no positive false alarm is indicated.
\end{abstract}

KEYWORDS: Concrete crack evaluation, Hybrid image scanning, Vision, Laser thermography, Nondestructive Testing

\section{INTRODUCTION (DETAILS FOR SUBMITTING EXTENDED ABSTRACT)}

Concrete crack evaluation is crucial for maintaining various civil infrastructures such as bridge, building, nuclear power plant, highway and so on. Visual inspection has been commonly used for their periodic inspection in the fields, but is it time-consuming, labor-intensive and expert-dependent [1]. To overcome the limitations, a number of nondestructive testing methods have been proposed. Ultrasonic methods have advantages of high sensitivity to cracks, high penetrating capability and non-invasive inspection [2,3]. However, they typically require sensor installation onto the concrete surface. Since ultrasonic waves can propagate only short distance along concrete structures, the limited spatial responses may not effective on large scale concrete structures. Moreover, complex signal processing is often necessary for crack identification because of the inhomogeneity of concrete. Another promising tool for concrete crack inspection is infrared (IR) thermography. The IR thermography enables to achieve noncontact inspection, simple operation and intuitive physical interpretation $[4,5]$. Recently, vision image processing methods integrated with an unmanned vehicle have been proposed [6, 7]. Although both IR thermography and vision images can provide meaningful crack information, they have own technical limitations. In the captured vision image, surface disturbances such as dust and undesired surface mark may produce positive false alarms. Furthermore, the capturing angle and shading effects often hinder precise crack evaluation, meaning the negative false alarm. On the other hand, the IR thermography has high crack detectability regardless of environmental variations if a precisely controlled heat source is equipped. However, surface pattern or joint on concrete structures are often recognized as cracks. In this study, the hybrid images between vision and IR images are used for concrete crack evaluation so that their technical limitations can mutually compensate each other.

\section{DEVELOPMENT OF THE HYBRID IMAGE SCANNING SYSTEM}

The hybrid image scanning system is composed of excitation, sensing and control units, as shown in Fig. 1. The continuous-wave (CW) laser emits a point laser beam, and the point laser beam is subsequently changed to a line shape beam through the line beam generator in the excitation unit, once the control signal is transmitted from the control unit to the heating and sensing units. Then, the line laser beam is exerted onto a target concrete surface of interest, generating thermal waves. Simultaneously, the vision and IR cameras in the sensing unit capture the corresponding responses. The entire region of interest (ROI) can be effectively inspected by spatially scanning the hybrid image scanning system. Here, the spatial scanning mechanism is necessary, because concrete 
materials typically have small thermal conductivity, and the vision and IR cameras have limited field of view (FOV). Note that the excitation and sensing units are all synchronized with the control unit as shown in Fig. 1.

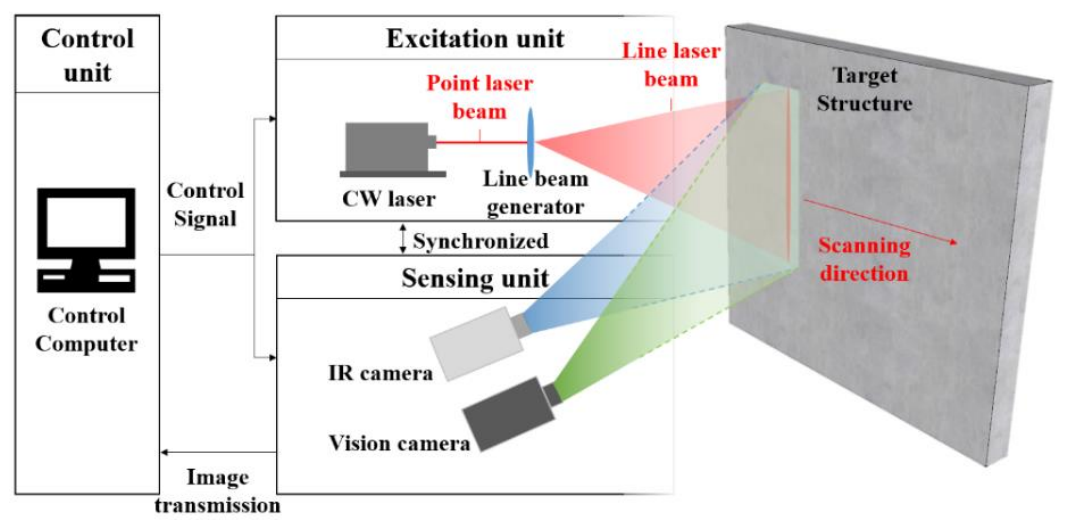

Fig. 1 Overview of the hybrid image scanning system.

Once the hybrid images are acquired using the hybrid image scanning system, data processing is subsequently performed. Since the hybrid image scanning system continuously moves along the target structure, the FOV of the vision and IR cameras is also continuously altered in the time and spatial domains. Therefore, the IR images need to be processed for precise crack evaluation as following three steps.

\section{Time-spatial-integrated coordinate transformation}

The raw vision and IR images with the time- and spatial-varying FOV are transformed to the spatially integrated ROI images as a function of time, which is defined as the $V^{R O I}$ and $I^{R O I}$ images, respectively. The $V^{R O I}$ and $I^{R O I}$ images are constructed using the following time-spatial-integrated coordinate transformation. Assume that the hybrid image scanning system spatially moves along only the $x$ direction.

$$
\left[\begin{array}{c}
x^{*} \\
y \\
t^{*}
\end{array}\right]=\left[\begin{array}{lll}
0 & 0 & 1 \\
0 & 1 & 0 \\
1 & 0 & 0
\end{array}\right]\left[\begin{array}{l}
x \\
y \\
z
\end{array}\right]
$$

where the $x, y$ and $z$ axes are the coordinates of the raw vision and IR images, and the $x^{*}, y$ and $t^{*}$ axes correspond to the $V^{R O I}$ and $I^{R O I}$ images. The $V^{R O I}$ images show the spatially integrated surface image, and the $I^{R O I}$ images can display the time-varying thermal responses in spatially fixed ROI. Here, $x *$ is the spatially integrated coordinate, and $t^{*}$ is the time duration right before and after the line laser beam heating.

\section{Phase mapping based on Hilbert transformation}

Now, the phase mapping process is subsequently performed only in the $I^{R O I}$ images for precise crack evaluation. If various size cracks exit in a single image, macro-cracks are typically overwhelmed while micro-cracks are hidden due to their amplitude difference. The phase mapping process overcomes the limitation related to the crack visibility issue, because the instantaneous phase information is physically self-normalized along each pixel. First, all pixel values of the $I^{R O I}$ images are transformed by Hilbert transformation along the $t^{*}$ axis [8].

$$
H\left(x^{*}, y, t^{*}\right)=\frac{1}{\pi} P \int_{-\infty}^{\infty} \frac{I^{R O I}\left(x^{*}, y, \tau\right)}{t^{*}-\tau} d \tau
$$

where $P$ stands for the Cauchy principle value of the integral. $H\left(x^{*}, y, t^{*}\right)$ mathematically gives the complex numbers. Then, the instantaneous phase value of each pixel can be calculated. Next, the phase image is constructed by cumulating all phase values along the $t^{*}$ axis.

\section{Spatial derivative}

The phase image obtained in the previous step gives the crack-induced phase difference information, but it still includes unwanted noise components. These noises can be removed by employing the spatial derivative along the scanning direction ( $x$-direction) of the phase image. The final processed image is coined as a $F$ image. 


\section{EXPERIMENTAL VALIDATION}

A target concrete specimen with $103 \mathrm{MPa}$ of compressive strength is prepared by mixing cement, silica sand, fly ash, superplasticizer and water. Then, artificial cracks are created in the concrete specimen by inserting $150 \mu \mathrm{m}$ acrylic slots. The artificial cracks can be divided into two types, i.e. macro-cracks $(\geq 500 \mu \mathrm{m})$ and micro-cracks $(<500 \mu \mathrm{m})$ depending on their width. In addition, a fake crack is introduced by using a pencil for the positive false alarm test. The CW laser (TMA-532-15T, TMA) generates a point laser beam with a wavelength of $532 \mathrm{~nm}$. The point laser beam is transformed into $250 \mathrm{~mm}-\mathrm{long}$ line laser beam using the line beam generator. The intensity of the line laser beam is set to approximately $37 \mathrm{~mW} / \mathrm{mm}^{2}$. The intensity of the line laser beam is optimized by considering the scanning speed of $23 \mathrm{~mm} / \mathrm{s}$ and the thermal conductivity of concrete $(0.8 \mathrm{~W} / \mathrm{m} \mathrm{K})$. The $\mathrm{CW}$ laser is $1600 \mathrm{~mm}$ apart from the concrete specimen. Then, the IR camera (A65, FLIR) measures the corresponding thermal waves in the time domain with a frame rate of $30 \mathrm{~Hz}$, a spectral range of $3 \mu \mathrm{m}$ to $5 \mu \mathrm{m}$, a pixel resolution of $640 \times 512$. The IR camera is $700 \mathrm{~mm}$ away from the concrete specimen, and the corresponding FOV has height, width and spatial resolution of $235 \mathrm{~mm}, 300 \mathrm{~mm}$, and $476 \mu \mathrm{m}$, respectively. The vision camera (GoPro Hero 4, GoPro) acquires the vision images in the time domain with a frame rate of $30 \mathrm{~Hz}$, a pixel resolution of $1080 \mathrm{x} 1920$. FOV of the vision camera is also same as the IR camera one. The specially designed scanning jig horizontally moves along the $x$ direction of ROI including fake, micro- and macro-cracks.

Once the raw vision and IR images are obtained by scanning the hybrid image scanning system, the $V^{R O I}$ and $I^{R O I}$ images can be constructed using the time-spatial-integrated coordinate transformation defined in Eq. (1). Then, the phase image is subsequently obtained using the $I^{R O I}$ images, but it still has a lot of noise components caused by the surface irregularity and irregular surface heating. Next, the noise component removal process is subsequently carried out, and it shows that both macro- and micro-cracks are well visualized. The crack visualization results are displayed in Fig. 2. Both $1 \mathrm{~mm}$-width macro-crack and $150 \mu \mathrm{m}$-width micro-crack are successfully evaluated as shown in Fig. 2 (a). Similarly, the almost closed-type crack having width of $1 \mu \mathrm{m}$ coexisting with the $600 \mu \mathrm{m}$ macro-crack is also well detected in Fig. 2 (b). On the other hand, one interesting fact to see in Fig. 2 (c) is that the fake crack is clearly shown in the $V^{R O I}$ image, while it is not highlighted in the $F$ image. Thus, it can be inferred that the proposed hybrid image scanning technique can be mutually complementary between vision and IR images, making it possible to minimize false alarms as their advantages retain.

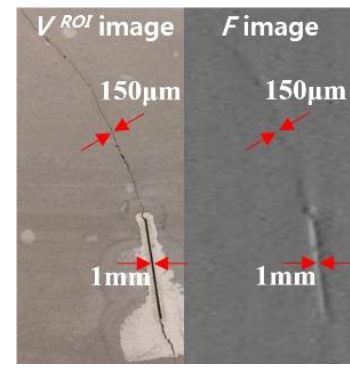

(a)

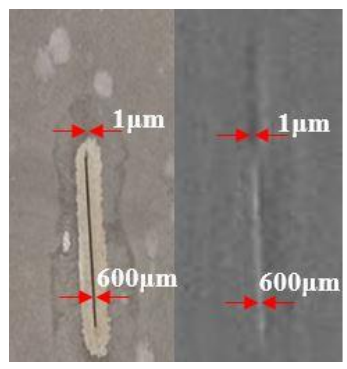

(b)

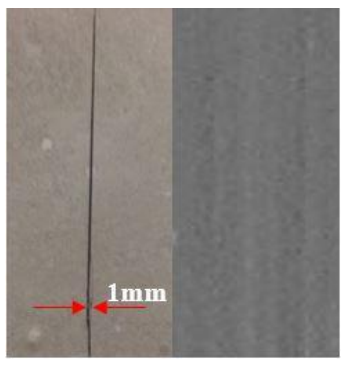

(c)

Fig. 2 Test results: (a) relatively large micro- and macro-cracks, (b) a closed-type crack with a macro-crack and (c) fake crack

\section{ACKNOWLEDGMENT}

This research was supported by a grant (16SCIP-C116873-01) from the Construction Technology Research Program funded by the Ministry of Land, Infrastructure and Transport of the Korean government.

\section{REFERENCES}

[1] Peter C. Chang, Alison Flatau, S. C. Liu, Review Paper: Health Monitoring of Civil Infrastructure, Structural Health Monitoring 2003; 2; 257.

[2] G. Song, $\mathrm{H} \mathrm{Gu}$, YL Mo, Smart aggregates: multi-functional sensors for concrete structures - a tutorial and a review, Smart Materials and Structures 17 (2008) 033001 (17pp)

[3] D.G. Aggelis, T. Shiotani, Repair evaluation of concrete cracks using surface and through-transmission wave measurements, Cement and Concrete Composites Volume 29, Issue 9, October 2007, Pages 700-711 
http://dx.doi.org/10.21611/qirt.2017.016

[4] M.R. Clark, D.M. McCann, M.C. Forde, Application of infrared thermography to the non-destructive testing of concrete and masonry bridges, NDT\&E International 36 (2003) 265-275

[5] T. Sakagami, S Kubo, Development of a new non-destructive testing technique for quantitative evaluations of delamination defects in concrete structures based on phase delay measurement using lock-in thermography, Infrared Physics \& Technology 43 (2002) 311-316.

[6] Najib Metni and Tarek Hamel, A UAV for bridge inspection: Visual servoing control law with orientation limits, Automation in Construction 17 (2007) 3-10.

[7] Jong-Woo Kim, Sung-Bae Kim, Jeong-Cheon Park, Jin-Won Nam, Development of Crack Detection System with Unmanned Aerial Vehicles and Digital Image Processing, Advances in Structural Engineering and Mechanics.

[8] Frank R. Kschischang, 'The Hilbert Transform' The Edward S. Rogers Sr. Department of Electrical and Computer Engineering University of Toronto, 2006 